\title{
Dermoscopy and methyl aminolevulinate: A study for detection and evaluation of field cancerization ir
}

\author{
A. Rossi a , V. Garelli a , G. Pranteda a , M. Cardone ${ }^{\text {a,* }}$, A. Anzalone a, M.C. Fortuna ${ }^{\text {a }}$, D. Di Nunno a , E. Mari ${ }^{\text {a }}$, \\ G. De Vita ${ }^{\text {b,c }}$, M. Carlesimo ${ }^{\mathrm{d}}$ \\ a Dipartimento di Medicina Interna e Specialità mediche Clinica Dermatologica, "Sapienza" Università di Roma, I Scuola, Italy \\ b Dipartimento di Fisica, Università della Calabria, ponte P. Bucci, cubo 31C, 87036 Rende, Italy \\ c CNR-Nanotec, UOS di Cosenza, ponte P. Bucci, cubo 31C, 87036 Rende, Italy \\ ' Dermatologia “Sapienza” Università di Roma, II Scuola, Italy
}

\section{A R T I C L E I N F O}

\section{Article history:}

Received 28 January 2016

Received in revised form 14 June 2016

Accepted 15 June 2016

Available online 16 June 2016

\section{Keywords:}

Dermoscopy

Actinic keratosis

Field cancerization

Methyl aminolevulinate

Fluorescence

Score

\begin{abstract}
A B S T R A C T
Actinic keratosis (AK) is a keratinocyte intraepidermal neoplasia UV light-induced that frequently appears in sun-exposed areas of the skin. Although historically AK was defined as "precancerous", actually it is considered as the earliest stage of squamous cell carcinoma (SCC) in situ. Since AKs can progress into invasive SCC, their treatment is recommended. AKs rarely develop as a single lesion; usually multiple lesions commonly affect an entire area of chronically actinic damaged skin. This has led to the concept of "field cancerization", an area chronically sun-exposed that surrounds peripherally visible lesions, in which are individualized subclinical alterations. One of the main principles endpoint in the management of AKs is the evaluation and the treatment of field cancerization. In this view, in order to detect and quantify field cancerization, we employed a method based on the topical application of methyl aminolevulinate (MAL) and the detection of the fluorescence emitted by its metabolite Protoporphyrin IX (PpIX); then, considering the extension and the intensity of measured fluorescence, we create a score of field cancerization. The results show that patients underwent to daylight PDT had a reduction of total score, from T0 to T2. Whereas in the group untreated we observed a stability of total score or a slightly worse. So, the method and the score used allows to evaluate with a good approximation the dimension of field cancerization and show the modification of it after treatment.
\end{abstract}

(c) 2016 Elsevier B.V. All rights reserved.

\section{Introduction}

Actinic keratosis (AK) is a common UV light-induced cutaneous lesion that results from the proliferation of atypical epidermal keratinocytes. The incidence of development of AK in Caucasian population increases with age, proximity to the equator and outdoor occupation. Australia has the highest skin cancer rate in the world. AKs are discovered in up to $40-50 \%$ of the Australian population older than 40 years. Although historically AK was defined as "precancerous" or "premalignant", in agreement with recent histopathologic and molecular studies it is considered as the earliest stage of squamous cell carcinoma (SCC) in situ [1-4]. Transformation of AK into invasive SCC happens following three progressive stages of keratinocyte intraepidermal neoplasia (KIN); in particular, KIN I and KIN II are characterized by

\footnotetext{
is Conflict of interest disclosure: None declared.

* Corresponding author at: Dipartimento di Medicina Interna e Specialità mediche Clinica Dermatologica, University of Rome "Sapienza” I School, V. le del Policlinico 155, 00161 Rome, Italy.

E-mail address: cardone_md@yahoo.it (M. Cardone).
}

proliferation of atypical keratinocytes in the epidermis lower third and in the lower two-third respectively, while KIN III is characterized by the presence of atypical keratinocytes in all layers of the epidermis. So, all AK lesions, regardless of intraepidermal neoplasia thickness, are potentially invasive [1] In according with the literature, the risk of progression of a single AK to an invasive SCC ranges from $0.25 \%$ to $20 \%$ per year and up to $60 \%$ of invasive squamous cell carcinoma arose from AKs [5-6]. The main risk factors for the development of AKs include: fair skin (Fitzpatrick type 1 or 2), old age, immunosuppressive therapy, PUVAtherapy and arsenic exposure. The most important cause of AK formation is UV-B radiation (wavelength $315-280 \mathrm{~nm}$ ) and UV-A radiation (400-315 nm) from sun-light, in fact AKs are typically seen on fairskinned people in chronic sun-exposed areas and their frequency correlates with cumulative UV exposure. The main mechanisms of AKs formation are genetic mutations which cause proliferation of altered keratinocytes, oxidative stress, immunosuppression, and altered apoptosis due to dysregulation of p53 pathways [5]. Clinically AKs appears at begin like small, rough spots that are easier felt than felt; afterwards the lesions enlarge, usually becoming red and scaly, often covered by yellow or brown adherent scales. Most lesions are only 3-10 mm, but 
they may enlarge to several centimeters in size; except in their hypertrophic form, the lesions show little or no infiltration. The surrounding areas may show evidence of widespread chronic sun damage with telangiectasia and yellowish discoloration [7]. Histologically AKs is characterized by dysplasia and architectural disorder of the epidermis, such as: abnormal keratinocytes of the basal layer with nuclear atypia, altered cellular polarity, hyperkeratosis and parakeratosis of the epidermis; an irregular acanthosis may be present [5]. Since AKs can progress into invasive SCC, their treatment is recommended. Approaches to AK can be broadly divided into lesion-directed (cryotherapy, laser therapy, surgery and curettage), or field-directed (diclofenac 3\%, imiquimod 5\%, 5fluorouracil, ingenol mebutate, chemical peels and ALA/MAL + PDT) [8]. Lesion-directed therapies are reserved for patients who have only a few isolated lesions and no elevated risk for development of invasive SCC. Field-directed therapies target both clinically visible lesions and preclinical alterations in the normal appearing skin surrounding the lesions; because AK is a visible marker of more extensive damage caused by chronic UV radiation exposure, the REAKT Working Group recommends field directed therapy as the optimal treatment approach for most patients [9]. The apparently normal skin which surround lesions is exposed to the same insults and could already reveal carcinoma-associated genetic alterations; this area is known as the 'field cancerization'. The concept of "field cancerization" was introduced by Slaughter in 1953 [10]. In recent molecular findings, it was established the following definition of field cancerization: "the presence of one or more areas consisting of epithelial cells that have genetic alterations. A field lesion (or shortly 'field') has a monoclonal origin, and does not show invasive growth and metastatic behavior, the hallmark criteria of cancer." A field lesion may have histological aberrations characteristic for dysplasia [11, $12]$. The field cancerization is an area chronically sun-exposed that surrounds peripherally visible lesions, in which are identified subclinical alterations detectable through different methods: histologically, molecular biology, confocal microscopy and through fluorescence [12,13]. One of the main principles endpoint in the management of AKs is the evaluation and the treatment of field cancerization. As the field cancerization is, for its definition, a subclinical lesion, it is not possible to recognize specific dermoscopy alterations, except those produced by chronic sun damage (telangiectasia, epidermis atrophy and yellowish discoloration, honeycomb pattern) [14]. Histologic features related to field cancerization are epidermal atrophy, increased pigmentation in basal keratinocytes, variable grade of atypia of basal keratinocytes, multiple vascular ectasias in the superior dermis, loss of normal keratinocyte polarization and intense degeneration of the dermic collagen with solar elastosis [15]. The biopsy for the evaluation of the field is invasive and not very useful, because it may not identify with precision the field of cancerization. Reflectance confocal microscopy (RCM) is a noninvasive imaging technique that allows the visualization of cellular and subcellular structures of the skin in vivo with near histological resolution. It was postulated that initial changes of epidermal morphology and cellular atypia may be observed by RCM before becoming clinically apparent. Therefore, RCM may be useful for the evaluation of actinic field cancerization and the detection of subclinical AK. The main features observed at RCM are: at the level of the stratum spinosum, discrete cellular and nuclear atypia of the keratinocytes, resulting in focal disruption of the epidermal architecture; at the level of the dermis, bright irregular bundles were observed, suggestive of solar elastosis. Furthermore, small, branched, bright structures morphologically corresponding to dilated blood vessels were seen [16]. Photodynamic therapy (PDT) with topical application of 5-aminolevulinic acid (ALA) or its methyl ester [methyl aminolevulinate (MAL)] as photosensitizers has proven to be clinically effective in AKs, Bowen's disease and superficial basal cell carcinoma (BCC) [17]. ALA or MAL is metabolized in Protoporphyrin IX (PpIX). All the cells own enzymes able to transform ALA to PpIX, although the altered cells with increased rate of replication and metabolism synthesize an excess of PpIX [18]. Preferential accumulation of PpIX in altered skin is used to treat various skin diseases. As PpIX has

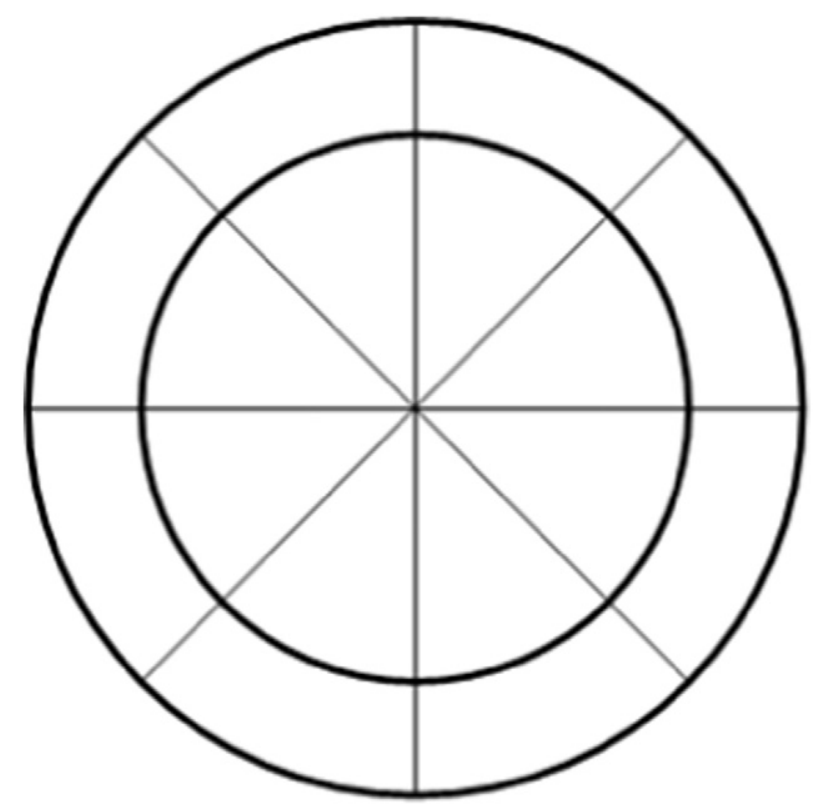

Fig. 1. Probe circular area.

fluorescence properties, its preferential accumulation in altered cells can be used as a marker of neoplastic keratinocytes. PpIX when excited by blue light $(408 \mathrm{~nm})$ shows red fluorescence. So, the field of cancerization could be identified by the detection of fluorescence emitted by PpIX accumulated in the keratinocytes [19] and the degree of fluorescence depends on the differential uptake of the photosensitizer by neoplastic cells [20].

\section{Materials and Methods}

In this paper we provide a method based on the topical application of MAL on AKs and perilesional areas and the successive detection of the fluorescence emitted by its metabolite (PpIX), in order to evaluate the score of field cancerization, considering the extension and the intensity of the recorded fluorescence. The method was applied to 20 patients (10 male and 10 female), affected by AKs on sun-exposed skin, aged between 58 and 80 years old with photo-type 2 and 3 . The exclusion criteria were: perilesional scarring and hypopigmentation, previous treatment on AKs and immunosuppression. Moreover, we considered as control group 10 patients not affected by clinically evident AKs, applying MAL on not sun-exposed skin. We provide an example of application and validity of this method for identifying the field cancerization surrounding the lesions through the fluorescence emitted by PpIX, a metabolite of MAL, which is a topical photosensitizer applied at the level of altered skin with a cream formulation containing $16 \%$ MAL (Metvix ${ }^{\circledR}$ ). The choice of MAL is due to greater selectivity, reduced time of occlusion and for the issuance of a more intense fluorescence for greater formation of PpIX than ALA. In the first visit (T0), patients signed the informed consent and have been subjected to clinical evaluation in
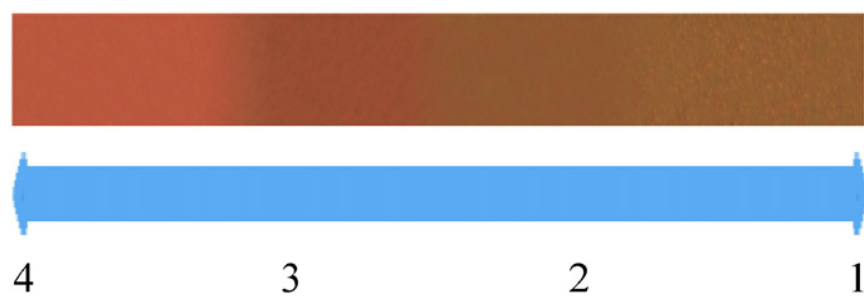

Fig. 2. Fluorescence scale. 
Table 1

Results of patients treated with daylight PDT.

\begin{tabular}{|c|c|c|c|c|c|c|c|c|}
\hline Patient & Age & Localization & $\begin{array}{l}\text { Extension } \\
\text { T0 }\end{array}$ & $\begin{array}{l}\text { Intensity } \\
\text { T0 }\end{array}$ & $\begin{array}{l}\text { Score } \\
\text { T0 }\end{array}$ & $\begin{array}{l}\text { Extension } \\
\text { T2 }\end{array}$ & $\begin{array}{l}\text { Intensity } \\
\mathrm{T} 2\end{array}$ & $\begin{array}{l}\text { Score } \\
\text { T2 }\end{array}$ \\
\hline 1 & 79 & Temple & 22 & 41 & 63 & 21 & 23 & 44 \\
\hline 2 & 65 & Temple & 18 & 24 & 42 & 10 & 11 & 21 \\
\hline 3 & 71 & Cheek & 21 & 24 & 45 & 13 & 14 & 27 \\
\hline 4 & 73 & Nose & 14 & 18 & 32 & 6 & 8 & 14 \\
\hline 5 & 54 & Scalp & 14 & 16 & 30 & 5 & 5 & 10 \\
\hline 6 & 71 & Cheek & 20 & 25 & 45 & 12 & 15 & 27 \\
\hline 7 & 58 & Scalp & 10 & 17 & 27 & 4 & 6 & 10 \\
\hline 8 & 66 & Temple & 20 & 22 & 42 & 9 & 11 & 20 \\
\hline 9 & 78 & Temple & 20 & 43 & 63 & 21 & 23 & 44 \\
\hline 10 & 70 & Nose & 12 & 19 & 31 & 7 & 7 & 14 \\
\hline
\end{tabular}

order to choose the area to be examined; after taking global clinical photographs of the affected areas, the lesions were examined using Dermoscope FotoFinder ${ }^{\circledR}$. Before the application of MAL, the patient's areas were cleaned with alcohol in order to facilitate the penetration of MAL; then, a $1 \mathrm{~mm}$ thin layer of cream with a concentration of $160 \mathrm{mg} / \mathrm{g}$ of MAL was accurately applied using wooden spatula on lesions and perilesional areas for an extension of $44 \mathrm{~cm}^{2}$. The treated areas were occluded using a plastic film as the first layer, followed by aluminum thin foil as the top layer, in order to avoid the early PpIX formation by the daylight. After an occlusion period of 100-120 min, the treated areas were uncovered and cleaned with gauze and saline solution. Then, we photographed and recorded the fluorescence intensity of PpIX emitted from the altered cells through the FotoFinderFluorescence ${ }^{\circledR}$, a probe testing an area of $44 \mathrm{~cm}^{2}$ and emitting a wavelength between $340 \mathrm{~nm}$ and $420 \mathrm{~nm}$. The pictures were acquired at a fixed distance of $10 \mathrm{~cm}$ from the patient. In order to achieve the highest fluorescence image quality while recording the images, all light sources in the room except the Wood's lamp of the probe were turned off or the probe was covered with dark cloth. The parameters evaluated by this method were extension and intensity of fluorescence, which is correlated with the intracellular concentration of PpIX. We divided probe circular area, sizing $44 \mathrm{~cm}^{2}$, into eight equal sectors and then split them at $r=R / \sqrt{2}$ into an inner circle and an external ring, so that each of the sixteen half-sectors has the same area (Fig. 1).

For each sectors, we considered two parameters: Extension of fluorescence $(0=$ absence of fluorescence, $1=$ presence of fluorescence in the central circle, $2=$ presence of fluorescence in the periferical circle) and Fluorescence Intensity (FI), established on the basis of scale gradation of red (1: low FI, 2: moderate FI, 3 high FI and 4: very high FI) (Fig. 2).

The total score is obtained adding the values of $\mathrm{E}$ and FI for each area and adding all the partial results. So, the total score ranges from 2 to 88 . In order to evaluate the sensitiveness of the method, we divided the 20 patients into two groups. The first group composed of 10 patients was treated with two sessions of daylight PDT at T0 and after one month (T1), while in the second one patients was not treated and kept the occlusion for $24 \mathrm{~h}$ with the aim to prevent the formation of PpIX. In order to highlight modification of the field cancerization after the treatment, patients come back to visit after three months (T2) and the score was calculated the score by measuring the fluorescence through FotoFinderFluorescence ${ }^{\circledR}$.

In Table 1-2 we show the collection of patient treated and untreated respectively. The localization of field cancerization and the respective parameter (extension, intensity and score) for both time T0 and T2 are shown.

In Table 3 we show the results of analysis, in particular the mean and standard deviation of the score variation $(<\mid \Delta$ Score $\mid> \pm \sigma)$ and of the variation normalized to the initial value $(<\mid \Delta$ Score/Score $(\mathrm{T} 0) \mid> \pm \sigma)$, as well as their minimum and maximum value.

\section{Results}

By a qualitative analysis, we observed a reduction of extension or intensity of fluorescence, clearly visible (Figs. 3-4).

Table 2

Results of untreated patients.

\begin{tabular}{|c|c|c|c|c|c|c|c|c|}
\hline Patient & Age & Localization & $\begin{array}{l}\text { Extension } \\
\text { T0 }\end{array}$ & $\begin{array}{l}\text { Intensity } \\
\text { T0 }\end{array}$ & $\begin{array}{l}\text { Score } \\
\text { T0 }\end{array}$ & $\begin{array}{l}\text { Extension } \\
\mathrm{T} 2\end{array}$ & $\begin{array}{l}\text { Intensity } \\
\mathrm{T} 2\end{array}$ & $\begin{array}{l}\text { Score } \\
\mathrm{T} 2\end{array}$ \\
\hline 1 & 73 & Cheek & 20 & 24 & 44 & 20 & 23 & 43 \\
\hline 2 & 58 & Forehead & 21 & 23 & 44 & 23 & 22 & 45 \\
\hline 3 & 80 & Temple & 15 & 15 & 30 & 17 & 14 & 31 \\
\hline 4 & 66 & Temple & 13 & 17 & 30 & 12 & 16 & 28 \\
\hline 5 & 65 & Forehead & 14 & 14 & 28 & 18 & 12 & 30 \\
\hline 6 & 74 & Forehead & 14 & 15 & 29 & 16 & 13 & 29 \\
\hline 7 & 64 & Cheek & 6 & 16 & 22 & 12 & 12 & 24 \\
\hline 8 & 65 & Forehead & 15 & 7 & 22 & 14 & 10 & 24 \\
\hline 9 & 58 & Temple & 12 & 46 & 58 & 20 & 43 & 63 \\
\hline 10 & 73 & Temple & 13 & 45 & 58 & 15 & 47 & 62 \\
\hline
\end{tabular}

Table 3

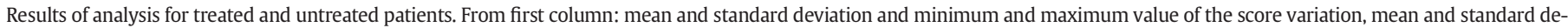
viation and minimum and maximum value of the variation normalized to the initial value.

\begin{tabular}{|c|c|c|c|c|}
\hline & $<\mid \Delta$ Score $\mid> \pm \sigma$ & $\Delta$ Score & $<\mid \Delta$ Score/score $(\mathrm{T} 0) \mid> \pm \sigma$ & $\Delta$ Score $/$ Score $(\mathrm{T} 0)$ \\
\hline Treated & $18.90 \pm 0.53$ & {$[17.00,22.00]$} & $0.48 \pm 0.04$ & {$[0.30,0.67]$} \\
\hline Untreated & $2.00 \pm 0.47$ & {$[0.00,5.00]$} & $0.06 \pm 0.01$ & {$[0.00,0.09]$} \\
\hline
\end{tabular}




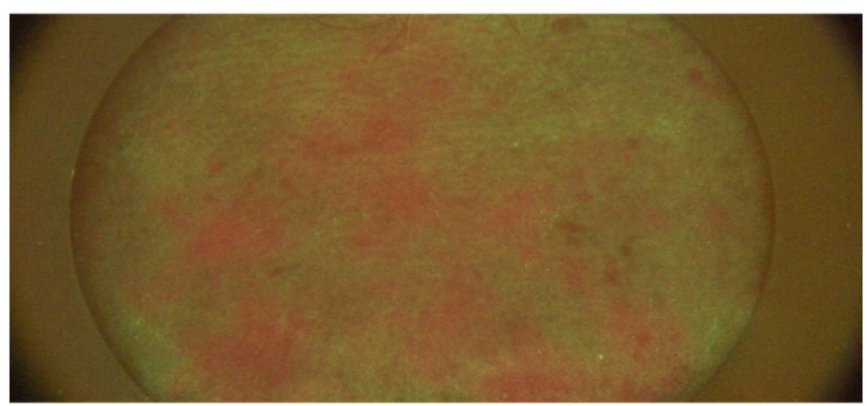

Fig. 3. Fluorescence TO in patient treated with PDT.

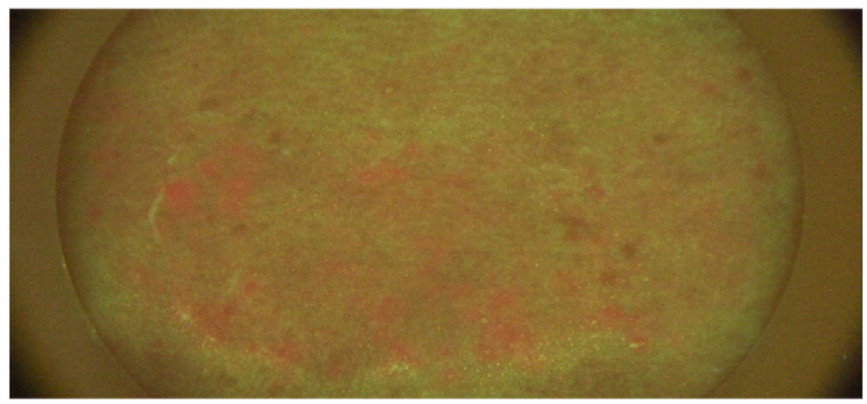

Fig. 4. Fluorescence T2 in patient treated with PDT.

Figs. 5-6 show the score of patient treated and untreated for T0 and $\mathrm{T} 2$ respectively. As can be seen in the Fig. 5 , the patients of the first group, treated with daylight PDT, had a reduction of the extension or intensity of fluorescence, with a consequent reduction of total score.

In order to quantify this reduction, we compute the mean and standard deviation of variation $\Delta$ Score $=|\operatorname{Score}(\mathrm{T} 2)-\mathrm{Score}(\mathrm{T} 0)|$ as listed in Table 3. Patients treated had a decrease of the average \langle|$\Delta$ Score $\mid>=$ 18.9. This value represents a significant \langle|$\Delta$ Score $/$ Score(T0) $\mid>=48 \%$ variation relative to score $\mathrm{T} 0$, and a nontrivial factor 40 in units of standard deviation $\sigma=0.5$.

On the contrary in the group untreated Fig. 6 we observed a stability of total score $(<\mid \Delta$ Score $|>=2.0,<| \Delta$ Score $/$ Score $(\mathrm{T} 0) \mid>=6 \%$ and a factor 4 in units of standard deviation $\sigma=0.5$ ).

In the control group the observation with Wood light showed a green fluorescence, that is expression of a non-pathological fluorescence, compatibly with skin without actinic damage.

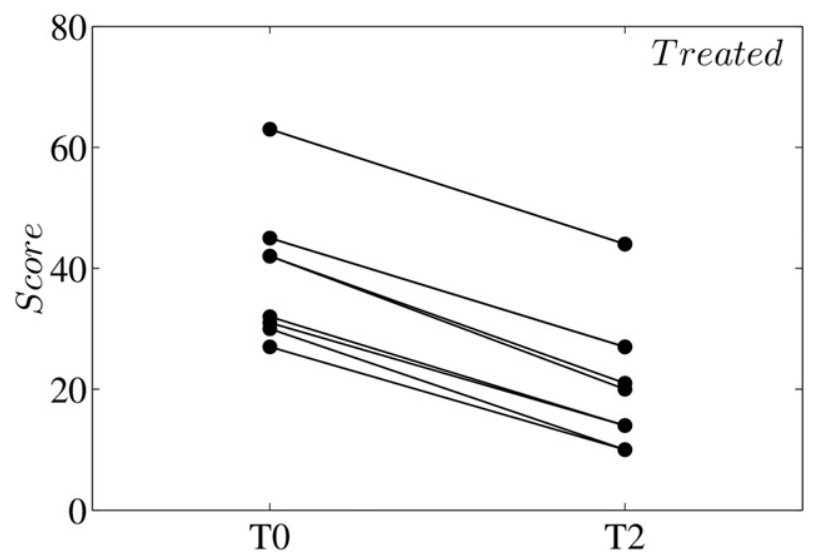

Fig. 5. Graphic illustrating patients treated with daylight PDT for T0 and T2.

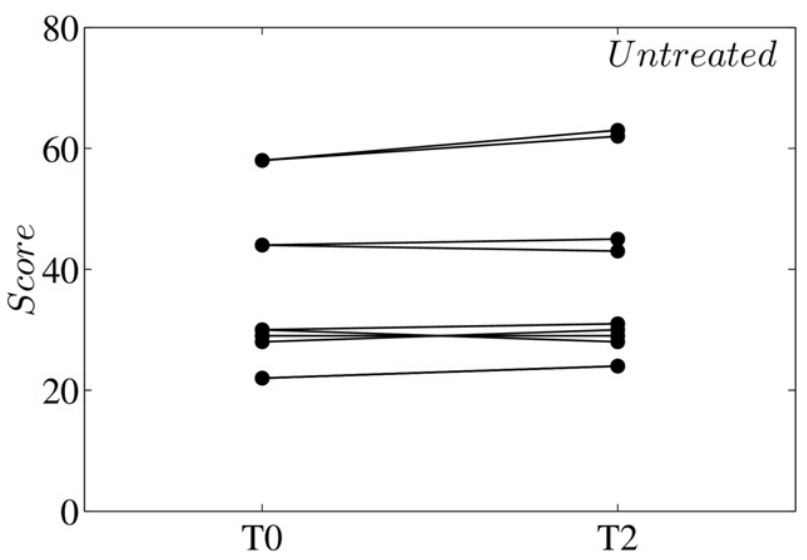

Fig. 6. Graphic illustrating patients untreated for $\mathrm{T} 0$ and $\mathrm{T} 2$.

\section{Discussion}

In this study we evaluated the effectiveness of a real-time, non-invasive method finalized to highlight the field cancerization based on fluorescence emitted by PpIX, a metabolite of MAL which is applied at the level of the skin altered. In addition to MAL's known therapeutic uses, the fluorescence emitted from its metabolite (PpIX) can be used as method to detection an area macroscopically and histologically normal but molecularly altered. For this purpose, we elaborated a score for quantification of field which could be useful not only in the diagnostic phase, but also for evaluate therapeutic response. The validity of the method is confirmed by the significant reduction $(<\mid \Delta$ Score $/ \mathrm{T} 0 \mid>=$ $48 \%$ ) of the score in patients treated with PDT and the lack of its significant variation in untreated patients $(<\mid \Delta$ Score/Score(T0) $\mid>=6 \%)$. An important element that emerged from our study is the greater expansion of the field of cancerization than $25 \mathrm{~cm}^{2}$ that are usually considered in the treatment field-directed.

\section{Conclusion}

Our method and the obtained score allows to evaluate noninvasively and with a good approximation the dimension of field cancerization and enables to verify its consequent disappearance or reduction after the treatment field-directed. The usual employment of the method could be useful to evaluate the efficacy of new drugs in clinical trial which have as endpoint their therapeutic activity on the field. Obviously the sensibility of the method could be not be compared with that of histopathology in the discrimination between SCC in situ and invasive SCC. At the best of our knowledge, this is the first study that propose the creation of a score for the individuation and evaluation of severity and extension of field of cancerization.

Nevertheless, the potential applicability of fluorescence in the detection and quantification of field cancerization requires further investigations.

\section{References}

[1] Fernández-Figueras MT1, C. Carrato, X. Sáenz, L. Puig, E. Musulen, C. Ferrándiz, A. Ariza, Actinic keratosis with atypical basal cells (AK I) is the most common lesion associated with invasive squamous cell carcinoma of the skin, J. Eur. Acad. Dermatol. Venereol. 29 (5) (2015) 991-997 (May).

[2] B.A. Lober, C.W. Lober, Actinic keratosis is squamous cell carcinoma, South. Med. J. 93 (7) (2000) 650-655.

[3] B.A. Ackerman, Solar keratosis is squamous cell carcinoma, Arch. Dermatol. 139 (9) (2003) 1216-1217.

[4] I. Zalaudek, S. Piana, E. Moscarella, et al., Morphologic grading and treatment of facial actinic keratosis, Clin. Dermatol. 32 (1) (2014) 80-87.

[5] J. Roewert-Huber, E. Stockfleth, H. Kerl, Pathology and pathobiology of actinic (solar) keratosis - an update, Br. J. Dermatol. 157 (Suppl. 2) (2007) 18-20 (Dec., Medline). 
[6] R. Marks, G. Rennie, T.S. Selwood, Malignant transformation of solar keratoses to squamous cell carcinoma, Lancet 1 (1988) 795-797.

[7] R. Anadolu-Brasie, A.R. Patel, S.S. Patel, et al., Squamous Cell Carcinoma of the Skin, McGraw-Hill, 2008.

[8] A. Dodds, A. Chia, S. Shumack, Actinic keratosis: rationale and management, Dermatol. Ther. 4 (1) (2014) 11-31 (Heidelb.).

[9] G. Hofbauer, M. Anliker, W.H. Boehncke, et al., Swiss clinical practice guidelines on field cancerization of the skin, Swiss Med. Wkly. 24 (144) (2014) w14026.

[10] D.P. Slaughter, H.W. Southwick, W. Smejkal, Field cancerization in oral stratified squamous epithelium, Cancer 6 (5) (1953) 963-968.

[11] B.J. Braakhuis, M.P. Tabor, J.A. Kummer, C.R. Leemans, R.H. Brakenhoff, A genetic explanation of Slaughter's concept of field cancerization: evidence and clinical implications, Cancer Res. (2003).

[12] E. Stockfleth, C. Ferrandiz, J.J. Grob, I. Leigh, H. Pehamberger, H. Kerl, Development of a treatment algorithm for actinic keratoses: a European Consensus, Eur. J. Dermatol. (2008).

[13] B.J. Braakhuis, C.R. Leemans, R.H. Brakenhoff, Expanding Fields of Genetically Altered Cells in Head and Neck Squamous Carcinogenesis, Semin, Cancer Biol, 2005.
[14] L.A. Ribeiro Torezan, C. Festa-Neto, Cutaneous Field Cancerization: Clinical, Histopathological and Therapeutic Aspects, An. Bras, Dermatol, 2013.

[15] J.A. Reed, C.R. Shea, Lentigo Maligna: Melanoma in Situ on Chronically Sun-damaged Skin, Arch. Pathol. Lab, Med, 2011.

[16] M. Ulrich, D. Krueger-Corcoran, J. Roewert-Huber, W. Sterry, E. Stockfleth, S. Astner Reflectance confocal microscopy for noninvasive monitoring of therapy and detection of subclinical actinic keratosis, Dermatology (2010).

[17] M.C. Almeida Issa, M. Manela-Azulay, Photodynamic Therapy: A Review of the Literature and Image Documentation, An. Bras, Dermatol, 2010.

[18] B.W. Henderson, T.J. Dougherty, How Does Photodynamic Therapy Work? Photochem, Photobiol, 1992.

[19] P. Babilas, S. Schreml, M. Landthaler, Rolf-Markus Szeimies, Photodynamic Therapy in Dermatology: State-of-the-art, Photodermatology, Photoimmunology \& Photomedicine, 2010.

[20] J.P. Ortonne, G. Gupta, N. Ortonne, L. Duteil, C. Queille, P. Mallefet, Effectiveness of cross polarized light and fluorescence diagnosis for detection of subclinical and clinical actinic keratosis during imiquimod treatment, Exp. Dermatol. (2010). 